\title{
Dust storm detection using random forests and physical-based approaches over the Middle East
}

\author{
Amir Hossein Souri ${ }^{1, *}$ and SAnaz VAJedian ${ }^{2}$ \\ ${ }^{1}$ Department of Earth and Atmospheric Sciences, University of Houston, Houston, TX 77204, USA. \\ ${ }^{2}$ Department of Surveying and Geomatics Engineering, University College of Engineering, University of Tehran, \\ North Kargar Ave., P.O. Box 11365-4563, Tehran, Iran. \\ ${ }^{*}$ Corresponding author.e-mail: asouri@uh.edu
}

Dust storms are important phenomena over large regions of the arid and semi-arid areas of the Middle East. Due to the influences of dust aerosols on climate and human daily activities, dust detection plays a crucial role in environmental and climatic studies. Detection of dust storms is critical to accurately understand dust, their properties and distribution. Currently, remotely sensed data such as MODIS (Moderate Resolution Imaging Spectroradiometer) with appropriate temporal and spectral resolutions have been widely used for this purpose. This paper investigates the capability of two physical-based methods, and random forests (RF) classifier, for the first time, to detect dust storms using MODIS imagery. Since the physical-based approaches are empirical, they suffer from certain drawbacks such as high variability of thresholds depending on the underlying surface. Therefore, classification-based approaches could be deployed as an alternative. In this paper, the most relevant bands are chosen based on the physical effects of the major classes, particularly dust, cloud and snow, on both emissive infrared and reflective bands. In order to verify the capability of the methods, OMAERUV AAOD (aerosol absorption optical depth) product from OMI (Ozone Monitoring Instrument) sensor is exploited. In addition, some small regions are selected manually to be considered as ground truth for measuring the probability of false detection (POFD) and probability of missing detection (POMD). The dust class generated by RF is consistent qualitatively with the location and extent of dust observed in OMERAUV and MODIS true colour images. Quantitatively, the dust classes generated for eight dust outbreaks in the Middle East are found to be accurate from $7 \%$ and $6 \%$ of POFD and POMD respectively. Moreover, results demonstrate the sound capability of RF in classifying dust plumes over both water and land simultaneously. The performance of the physical-based approaches is found weaker than RF due to empirical thresholds that are not always true.

\section{Introduction}

Dust storms usually are stimulated in arid and semi-arid regions with low annual rainfall, and can carry a large quantity of dust particles like an overwhelming tide to destroy crops, reduce visibility, ruin the communication facilities, and impact weather and climate (Nicholson 2000; James et al. 2007 ). To study these effects, regular monitoring of their distribution including sources and transport

Keywords. Random forests; dust detection; MODIS; decision tree.

Supplementary data pertaining to this article are available on the Journal of Earth System Science Website at http://www.ias.ac.in/jess/jul2015/supp/Amir.pdf 
is necessary. Remote sensing offers an exceptional capability to observe the spatial and temporal variability of dust. Dust events have been detected using TOMS (Total Ozone Mapping Spectrometer) and OMI (Ozone Monitoring Instrument) (Alpert et al. 2004), METEOSAT (Moorthy et al. 2007), AVHRR (Advances Very High Resolution Radiometer) (Evan et al. 2006; Zhu et al. 2007), and GOES-VISSR (Geostationary Operational Environmental Satellite, Visible Infra-Red SpinScan Radiometer) (MacKinnon et al. 1996). Due to high spatial coverage, temporal and spectral resolutions of MODIS (Moderate Resolution Imaging Spectroradiometer) and its imagery have been helpful when it comes to researching on dust phenomenon (Badarinath et al. 2007; Zha and Li 2007; Kaskaoutis et al. 2008; McGowan and Clark 2008).

Ackerman (1989) stated that the quantity of dust particles could be evaluated by brightness temperature difference (BTD) $(3.7 \mu \mathrm{m}, 11 \mu \mathrm{m})$. He improved his former work by additional bands of 8 and $12 \mu \mathrm{m}$ to perform dust detection using AVHRR and MODIS imagery (Ackerman 1997). According to the spectral characteristics of reflection of cloud, snow and water that reaches approximately a maximum at blue band and a minimum at $2.13 \mu \mathrm{m}$, and the reflection of dust which is similar to soil increasing with the reflective wavelengths, normalized the difference of these two bands (i.e., 0.469 and $2.13 \mu \mathrm{m}$ ) have a potential to distinguish snow, water and cloud from dust. Accordingly, an index known as Normalized Difference Dust Index (NDDI) was introduced in $\mathrm{Qu}$ and Kafatos (2006). Hao and Qu (2007) proposed Thermalinfrared Dust Index (TDI) for retrieving Aerosol Optical Thickness (AOT) using MODIS imagery. This index was calculated by exploiting four emissive infrared bands. Xie (2009) proposed a decision rule-based method for observing and monitoring dust aerosols over both bright and dark surfaces by combining measurements of MODIS emissive and reflective bands. Zhao et al. (2010) suggested another algorithm of dust detection using satellite multi-channel images. The method can be applied to any multi-channel imagery with channels at (or close to) $0.47,0.64,0.86,1.38,3.9,11.0$, and $12.0 \mu \mathrm{m}$. The two latter methods will be outlined in detail in next sections.

Due to distinct radiative signatures of dust in the UV, visible, and IR channels, most studies have focused on the physical-based approaches. Although physical-based methodologies are directly linked with spectral characteristics, they generally suffer from drawbacks such as empirical thresholds depending on the underlying surface that cannot be applied to all images. The shortcomings lead to the fact that the ability of dust detection can differ from location to location. Therefore, it should be taken into account that the answer of a test should not be an index, rather a confidence indicator between 0 and 1 , where 1 denotes a high confidence in dusty conditions. The output can be obtained by statistical methods. Because of the drawbacks of physicalbased approaches, in recent years, there has been an increased interest in methods that approach the problem of dust detection through statistical procedures. Little work has been done on the statistical-based dust detection approaches. RivasPerea et al. (2010) developed a Maximum Likelihood Classifier (MLC) and a Probabilistic Neural Network (PNN) to automate dust storm detection process. Their results indicated that PNN provided improved classification performance with reference to MLC. Moreover, in Chacon-Murguía et al. (2011), an artificial neural network (ANN) was proposed to identify not just dusty areas, but also vegetation and soil. The main drawback of their work is using MODIS cloud mask product. MODIS cloud mask cannot be used due to the fact that it has a tendency to misclassify thick dust as cloud (Martins et al. 2002; Frey et al. 2008).

The aim of this study is to evaluate the capability of Random Forests (RF) (Breiman 2001) classifier, for the first time in the field of dust detection, in comparison to two physical-based methods. This paper is presented in five sections. Section 2 describes data used and feature selection. In section 3, the detail of two physical-based algorithms proposed by Xie (2009), Zhao et al. (2010) and RF classifier are described. Section 4 describes the results of eight dust storms detected. The final section remarks on the major conclusions drawn from this research.

\section{Imagery data used}

\subsection{MODIS imagery}

The MODIS acquires global data daily in 36 spectral bands from $0.4-14.4 \mu \mathrm{m} ; 29$ bands with $1 \mathrm{~km}$, 5 with $500 \mathrm{~m}$, and 2 with $250 \mathrm{~m}$ nadir resolution (Salomonson et al. 1989). We read the latitude and longitude files associated with each MODIS scene and included in the Hierarchical Data Format (HDF) files provided by NASA Distributed Data Archive Center (DAAC) database. The resulting geocoded image was projected in latitude/longitude coordinated and corrected for the bow-tie effect (i.e., overlap of the satellite scan lines which produces a data repetition) by MODIS Reprojection Tool Swath (available at https://lpdaac.usgs.gov/tools/modis_reprojection_ tool_swath). Only AQUA imagery is used in this study, since OMI sensor is few minutes behind AQUA sensor. The selected bands for RF algorithm are listed in table 1. 
Table 1. Selected bands of MODIS.

\begin{tabular}{lr}
\hline Band number & $\lambda(\mu \mathrm{m})$ \\
\hline 1 & 0.645 \\
2 & 0.845 \\
3 & 0.469 \\
4 & 0.555 \\
6 & 1.640 \\
7 & 2.130 \\
8 & 0.412 \\
9 & 0.433 \\
20 & 3.750 \\
26 & 1.375 \\
29 & 8.550 \\
31 & 11.030 \\
\hline
\end{tabular}

Since expanding number of bands with no contribution to new information leads to degradation of classification accuracy, only the relevant bands based on the physical effects of main classes on both radiative and reflective bands are chosen. In addition, $\mathrm{RF}$ provides a statistical approach (Breiman and Cutler 2008) to measure the importance of the features. Both procedures are done to assure that the chosen bands can provide the most relevant and practical attributes.

Clouds are made of microscopic droplets of liquid water (water/warm clouds), tiny crystals of ice (ice clouds), or both (mixed phase clouds). Fog and low-altitude (up to about $3000 \mathrm{~m}$ ) cloud typically compose of water droplets, having modal radii 10-50 $\mu \mathrm{m}$ (Rees 2001). The particles in liquid water clouds can be well approximated as liquid spheres, and their optical properties can be computed using the Lorenz-Mie theory (Mie 1908). The theory implies that scattering is dominant at visible and near-infrared wavelengths. Therefore, using visible and near-infrared bands is the key for cloud detection particularly over water. Because dust absorbs at blue wavelength and appears visually brown, a band at $0.47 \mu \mathrm{m}$ for dust detection can be used. Also, a reflective band at $0.86 \mu \mathrm{m}$ for the detection of aerosols and vegetation can be applied. The spectral signature of water cloud and snow is very close in visible and near-infrared regions, because ice and water have similar refractive indices, but ice is slightly more absorptive from 1.55 to $1.7 \mu \mathrm{m}$. Therefore, $1.64 \mu \mathrm{m}$ is another feature to discriminate snow and ice clouds from warm clouds. It is usually problematic to detect and analyze optically thin clouds at visible or near-infrared wavelengths with an exception of $1.38 \mu \mathrm{m}$ that is particularly effective in retrieving thin cirrus reflectance (Gao et al. 2002). There is an advantage of exploiting $2.1 \mu \mathrm{m}$ to detect dust especially from high clouds, snow and water, because it has high values in dusty regions. The unique spectral properties of dust in the TIR region, especially the ' $\mathrm{V}$ ' shape signature of silicate-based absorber in the 8.3-12.0 $\mu \mathrm{m}$ region, makes it possible to identify dust with IR measurements (Ackerman 1989, 1997; Roskovensky and Liou 2005). Moreover, thin clouds are important in the IR region because of strong absorption. Regarding the specification of clouds that makes their temperatures reside between $40^{\circ}$ and $0^{\circ} \mathrm{C}$ (Yang et al. 2003) and concerning dust that might be colder than underlying surface (especially over desert); $11 \mu \mathrm{m}$ might be used for cloud and dust detection over land. According to the spectral variability of dust emissivity at 3.75 and 8.55 $\mu \mathrm{m}$, band 20 (i.e., $3.75 \mu \mathrm{m}$ ) and band 29 (i.e., $8.55 \mu \mathrm{m})$ are used. Retrieving dust plumes over a bright surface such as arid and semi-arid regions is a challenge (Hsu et al. 2004). Even though in these regions the surface reflectance is typically too bright in visible and near-infrared bands, they are much darker in deep blue bands. Therefore, these bands are exceptional features to retrieve dusty pixels (and their properties) over deserts due to high contrast between a dark background and bright dusty pixels.

In this research, eight datasets of AQUA imagery taken on $25 / 01 / 2005,19 / 04 / 2005,05 / 07 / 2005$, 17/09/2008, 25/01/2010, 11/05/2010, 30/06/2011 and 20/06/2012 from different parts of the Middle East are used.

\subsection{OMI imagery}

In order to assess the accuracy of results, OMAERUV product from OMI sensor is used. OMI sensor measures radiance in the wavelength range $264-504 \mathrm{~nm}$ with $13 \times 24 \mathrm{~km}$ nadir resolution. Because of the high UV absorption of hematite and other iron-based components of desert dust, the near-UV measurement is suitable for the detection and quantification of dust (Torres et al. 2007). OMI on-board EOS AURA and AQUA spacecrafts are operated in the A-train orbit. Accordingly, concerning dust storms moving fast in a short timeperiod, OMI is an appropriate sensor to validate AQUA results. In this study, OMAERUV AAOD (Aerosol Absorption Optical Depth) product is used because of very low reflectance of all terrestrial surfaces in the UV, including the arid and semi-arid regions that appear very bright in the visible and near-IR regions. AAOD is a dimensionless measure of the absorption. High values of AAOD explain more presence of dust. Torres et al. (2007) pointed out that OMAERUV was more reliable over land than water, and by the presence of sub-pixel cloud contaminations AAOD became less accurate. It is worth mentioning that, although, MODIS AOT product is available with the scenes considered in this paper, it has gaps in bright areas 
(e.g., sun glint region over ocean and desert over land); so this study only used OMAERUV AAOD product.

\section{Methodology}

In order to perform dust detection, three methods are used. The first two methods are physicalbased and the third one is classification-based. The physical-based methods are decision-tree methods proposed by Xie (2009) and Zhao et al. (2010). The classification-based method (i.e., random forests) is used for the first time in the field of dust detection.

\subsection{Xie's method}

By combining measurements of MODIS emissive and reflective bands, Xie (2009) proposed a decisionrule based method for observing and monitoring dust aerosols over both bright and dark surfaces. Using MODIS true-colour image, Xie identified correctly the core part of dust pixels except for some missed pixels which contained a relatively weak dust signal. The results were not only consistent with human eyes feedback, but also with Cloud-Aerosol Lidar with Orthogonal Polarization (CALIOP) Vertical Feature Mask (VFM) results. More than 90\% dusty pixels were identified accurately with reference to CALIPSO VFM. The algorithm also claims to work efficiently in areas which are close to clouds or mixed with clouds. Four thresholds were used in the method. The first two thresholds were for extracting cloud, snow and water and the last two thresholds were for distinguishing dusty regions from soil and vegetation.

The NDDI is the first feature used. It normalizes difference of bands $7(2.1 \mu \mathrm{m})$ and $3(0.47$ $\mu \mathrm{m})$ in MODIS sensor. As mentioned before, the index is suitable for separating snow, water and cloud from dust and surface classes. According to the spectral characteristics of the classes, NDDI is positive for dust and surface, and negative for others. The next feature is BTD $(11 \mu \mathrm{m}$ and 12 $\mu \mathrm{m})$. This feature apparently is negative for cloud and positive for dusty regions over land; however, this condition might be false for low level clouds (towering cumulus) and dust over water (Zhao et al. 2010). Both dust and surface scenes have similar brightness temperature (BT) at band 20 (3.7 $\mu \mathrm{m})$; but dust has much lower BT at long wave infrared (LWIR) than surface. Among bands in LWIR, BTD of 3.7 and $11 \mu \mathrm{m}$ bands was used due to the largest difference between dust and surface scenes. Furthermore, the logarithm of reflectance in red band was exploited to separate dust from surface scenes in the method. Using logarithm of red band could enhance the resolving power of this index, especially in small reflectance range (Xie 2009). The flowchart of the algorithm is shown in figure 1. The first statement including NDDI and BTD (11 and $12 \mu \mathrm{m}$ ) is used for filtering out cloud, water and snow. The remaining pixels are then divided into two surface types: bright surface (e.g., soil) and dark surface (e.g., vegetation). This pre-stored information is regarded as a problem in this method. After performing the last two thresholds, $3 \times 3$ majority filter can be carried out on the resultant classes in order to remove noisy (discrete) pixels.

\subsection{Zhao's method}

Zhao et al. (2010) proposed an algorithm for detection of dust during daytime. The algorithm enables dust detection over both land and ocean for any sensors measuring at (or close to) 0.47, 0.64, 0.86, $1.38,3.9,11.0$, and $12.0 \mu \mathrm{m}$. The method exploits spectral and spatial empirical tests along with some uniformity texture examinations. The results obtained from this approach was consistent with MODIS AOT product (Zhao et al. 2010). However due to relatively weak aerosol signal associated with a bright surface, the dust detection algorithm neglected thin dust (Zhao et al. 2010). It also did not work over snow/ice or high ice clouds (Zhao et al. 2010).

The variables used for this approach were defined as follows (wavelengths are given in subscripts):

- MeanR: mean of reflectance for $3 \times 3$ pixels, $\left(\mathrm{MeanR}_{0.86 \mu \mathrm{m}}\right)$

- StdR: standard deviation of reflectance for $3 \times 3$ pixels $\left(\operatorname{StdR}_{0.86 \mu \mathrm{m}}\right)$

- $\mathrm{Rat}_{1}=\left(\mathrm{R}_{0.64 \mu \mathrm{m}}-\mathrm{R}_{0.47 \mu \mathrm{m}}\right) /\left(\mathrm{R}_{0.64 \mu \mathrm{m}}+\mathrm{R}_{0.47 \mu \mathrm{m}}\right)$

- $\mathrm{Rat}_{2}=\left(\mathrm{Rat}_{1} \times \mathrm{Rat}_{1}\right) /\left(\mathrm{R}_{0.47 \mu \mathrm{m}} \times \mathrm{R}_{0.47 \mu \mathrm{m}}\right)$

- $\mathrm{NDVI}=\left(\mathrm{R}_{0.86 \mu \mathrm{m}}-\mathrm{R}_{0.64 \mu \mathrm{m}}\right) /\left(\mathrm{R}_{0.86 \mu \mathrm{m}}+\mathrm{R}_{0.64 \mu \mathrm{m}}\right)$

- $\mathrm{MNDVI}=\mathrm{NDVI}^{2} /\left(\mathrm{R}_{0.64 \mu \mathrm{m}} \times \mathrm{R}_{0.64 \mu \mathrm{m}}\right)$

\subsubsection{Dust detection over land}

The detection of dust over land involves four group tests. The first one is for removing dead (or bad) pixels. The second group filters out water cloudy pixels. As mentioned in previous approach, BTD (11 and $12 \mu \mathrm{m})$ becomes more positive with increasing dust loads; however, low level clouds can also have a positive BTD. Therefore, two additional conditions were applied to remove water cloudy pixels. Dust reflects solar BT radiation at $3.9 \mu \mathrm{m}$ more than that at $11 \mu \mathrm{m}$. Therefore, a large BTD $(3.9$ and $11 \mu \mathrm{m})$ might flag pixels as dust. In 


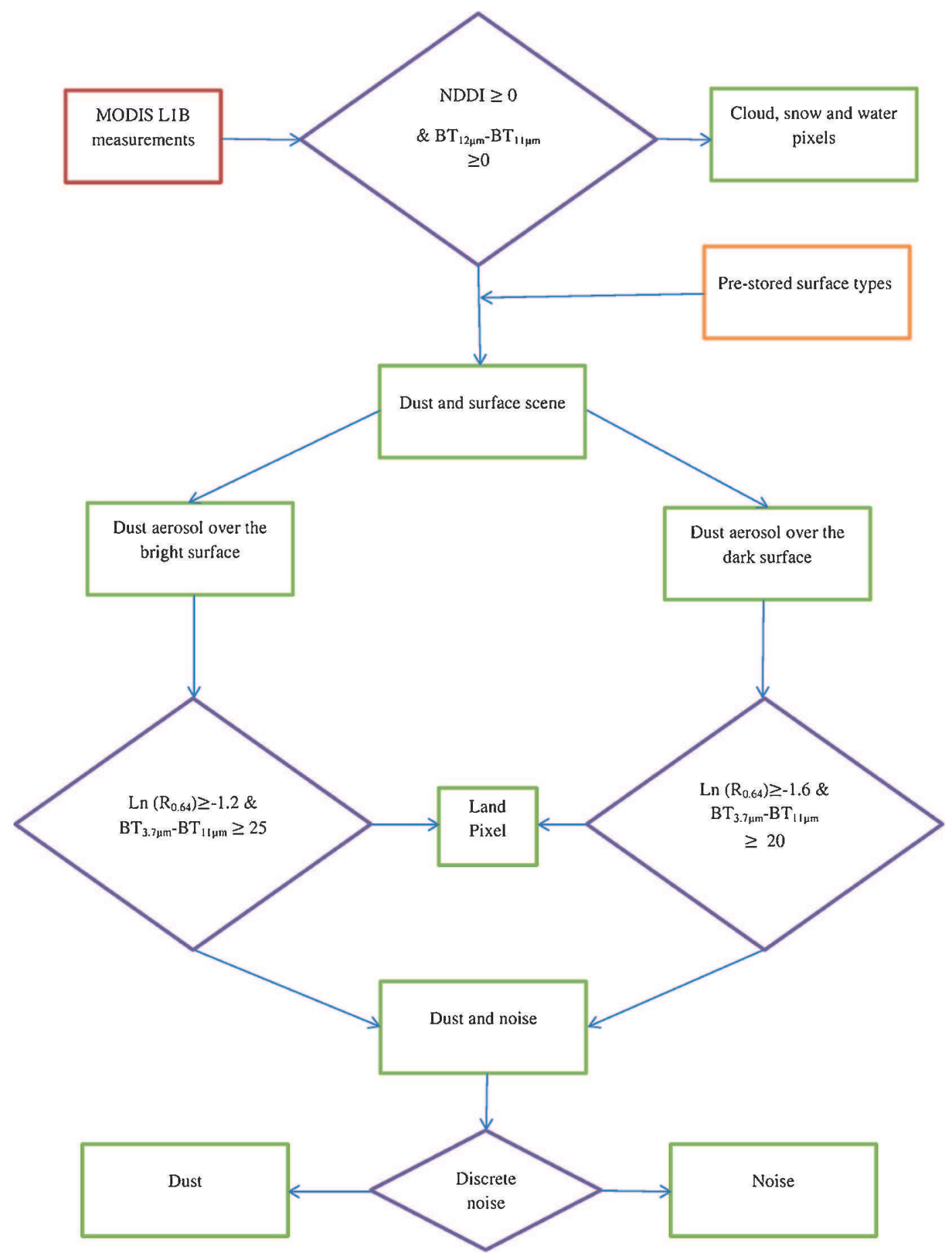

Figure 1. The flowchart of Xie's method.

addition, because of absorbing blue wavelength by dust, MNDVI and Rat ${ }_{2}$ were used for the detection over semi-arid surfaces with small amount of vegetation (Zhao et al. 2010). The last group tests are for revealing optically thick dust storms. Generally MNDVI values for cloud and clear-sky are larger than 0.2 but for dusty pixels (especially thick dust) is less than this value. The flowchart of the approach over land is shown in figure 2 .

\subsubsection{Dust detection over ocean}

The detection of dust over water also involves four group tests. The first and second groups were again proposed for screening out dead and water cloudy pixels, respectively. The uniform distribution of dust over ocean compared to cloud required a texture examination (i.e., StdR). Due to less reflection from dust than cloud over ocean, a reflective test 


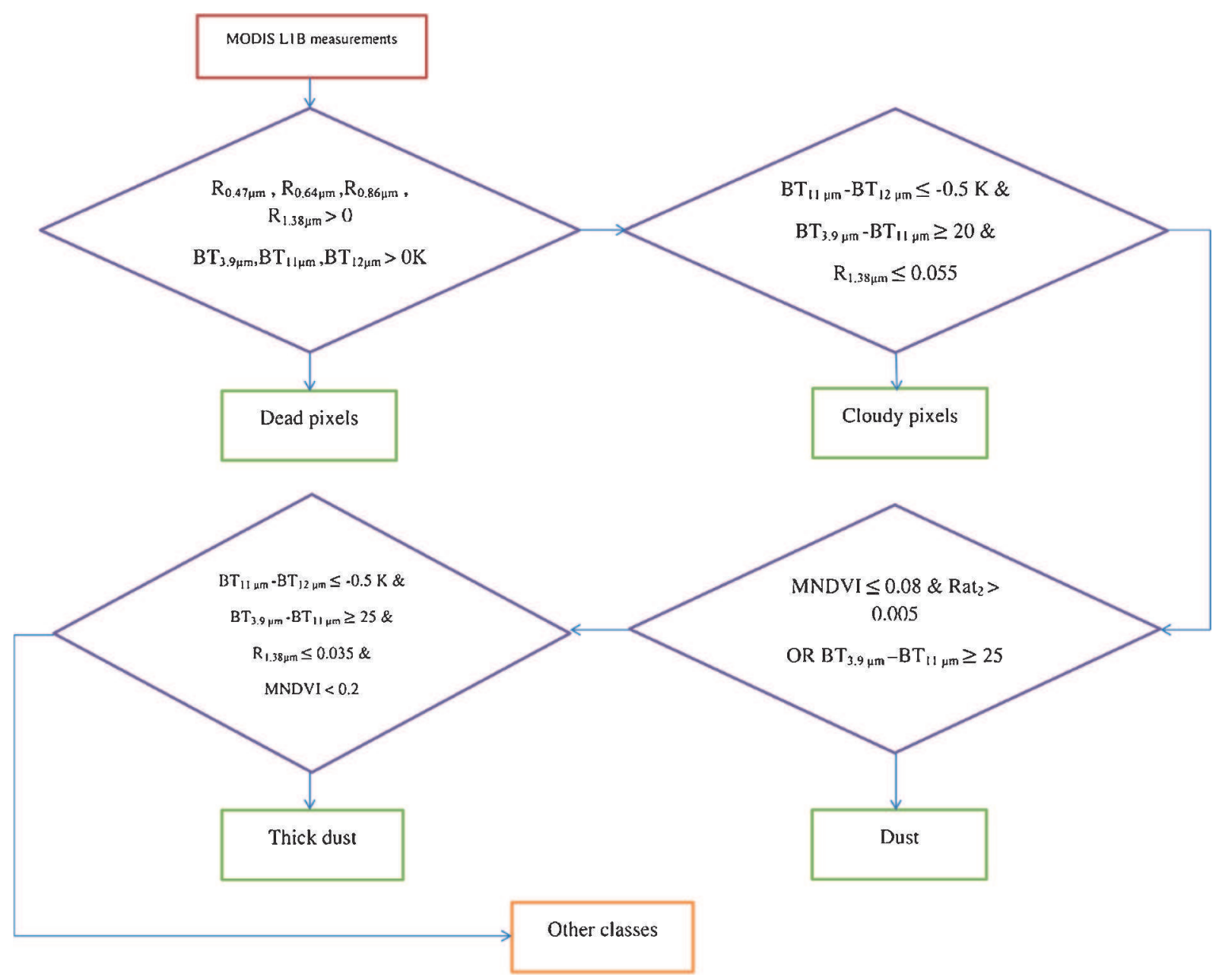

Figure 2. The flowchart of Zhao's method for dust detection over land.

was added to BTD $(3.9,11 \mu \mathrm{m})$ test. There are four separate tests for dust detection over water; each pixel satisfying any of the three tests is labelled dust class. According to the research of Ackerman (1997) for detecting airborne dust using BTD (11 and $12 \mu \mathrm{m}$ ), this BTD was also used for dust detection over water. However, BTD (11 and $12 \mu \mathrm{m})$ may show false detection. Therefore, to obtain better results, NDVI was coupled to the methodology. The attenuation coefficient (sum of scattering and absorption coefficients) of aerosols at optical and infrared wavelengths is dominated by scattering rather than absorption (Rees 2001). This value is usually approximated by the angstrom relation (Rees 2001):

$$
\gamma=\gamma_{0} \lambda^{-n}
$$

where $\gamma$ and $\lambda$ are attenuation coefficient and wavelength, respectively. For aerosols, the value of $n$ is approximately from 1-2. According to equation (1), by decreasing the wavelength, scattering increases. As a result, $\mathrm{R}_{0.86 \mu \mathrm{m}} / \mathrm{R}_{0.64 \mu \mathrm{m}}$ ratio was used in this approach for flagging dust plumes over water. Similar to dust detection over land, the last group test is for detecting heavy dust. The flowchart of this approach is depicted in figure 3 .

\subsection{Random Forests Classifier}

Random Forests Classifiers are machine learning algorithms. Instead of one classifier, they are constructed from a set of classifiers. The algorithms called ensemble classification can extensively improve the performance of learning. The most well-known ensemble classifiers are Boosting, Bagging and RF. The Bagging algorithm (Bootstrap aggregating) (Breiman 1996) votes classifiers results generated by different bootstraps. A bootstrap sample is generated by uniformly sampling $n$ instances from the training set with replacement (Efron and Tibshirani 1993). $N$ bootstrap samples are generated and a classifier $T_{i}$ is formed from each bootstrap sample. Simple majority vote is taken for prediction which means a final classifier $T^{*}$ is built from $T_{1}, T_{2}, \ldots, T_{N}$ whose output is the class most often predicted. Similar to Bagging, the Boosting algorithm generates a set of classifiers and votes them. However, Boosting algorithm 


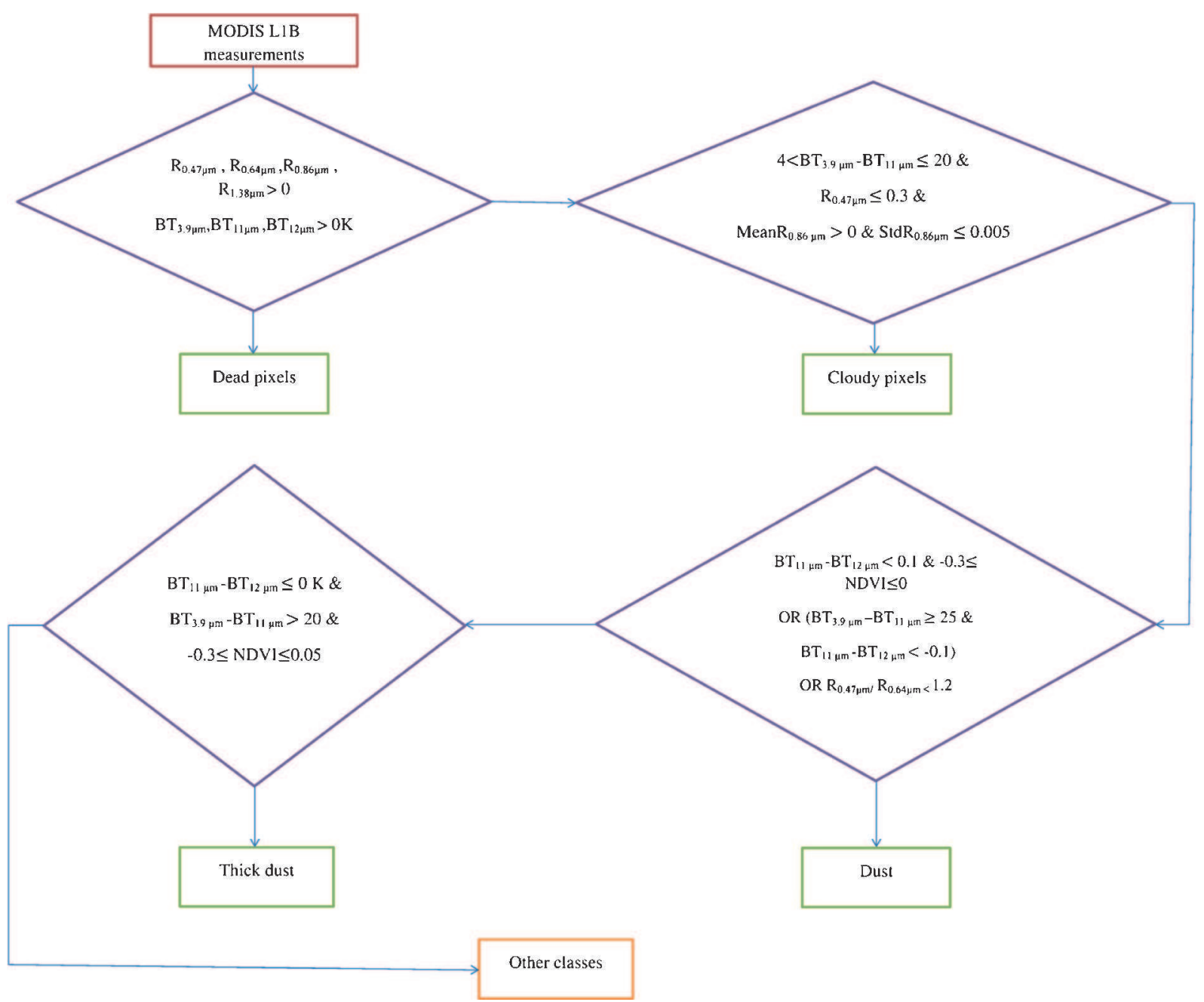

Figure 3. The flowchart of Zhao's method for dust detection over water.

generates the classifiers sequentially, while Bagging generates them in parallel. Boosting also alters the weights of the training samples. AdaBoost (Freund and Schapire 1997) is the most representative algorithm in this family. AdaBoost is known to reduce both bias and variance (Friedman et al. 1998) and similarly, Support Vector Machine (SVM) boosts the margins (Rudin et al. 2004). However, the main limitations of boosting are noise sensitivity, time-consuming and over-fitting (Gislason et al. 2006).

$\mathrm{RF}$ is the most effective ensemble method in respect to accuracy compared to AdaBoost, SVM, discriminant analysis and ANN (Artificial Neural Networks) (Liaw and Wiener 2002; Breiman and Cutler 2005). RF constructs a collection of tree-structured classifiers using Classification And Regression Tree (CART) algorithm (Breiman 2001), and it is an advanced version of Bagging such that randomness is added to it. The training set for growing each tree is $N$ sample at random with replacement from the original data. In
Bagging algorithm, for a given bootstrap sample, an instance in the training set has probability $1-$ $(1-1 / n)^{n}$ of being selected at least once in the $n$ times samples from the training set. For large $n$, this is about $1-1 / \mathrm{e}=63.2 \%$. It means $36.8 \%$ of instances are left out and they do not take part in construction of the tree. In RF, these instances, called Out-Of-Bag (OOB) are used to calibrate the performance of each tree. Therefore, there is no need for cross validation (CV) or separate test set to estimate the strength and correlation of trees and the error of predictions. An unpruned tree from each bootstrap sample is grown such that at each node of the tree the splitting attribute is selected from a randomly chosen sample of attributes. It was suggested in Breiman (2002) that by setting the number of attributes $(r)$ to the square root of number of overall features, optimum results might be gained. At each node, splitting is performed according to a criterion in CART algorithm. In this study, GINI index is utilized to accomplish splitting. The attribute with the highest GINI index is 
chosen for splitting in that node. The GINI index can be written as (Raileanu and Stoffel 2004):

$$
\text { GINI }=1-\sum_{c} p^{2}(c \mid t)
$$

where $c$ is the number of classes, $t$ is node of a tree and $p$ is relative frequency of $c$. Equation (2) means that if all the samples are on the same class, the impurity is zero, otherwise it is of positive value. Two parameters must be specified to start RF algorithm: $N$ (number of trees to grow) and $m$ (the number of attributes). The strategy of RF makes it fast and efficient on large databases. It is robust against over-fitting and coped with 'curse of dimensionality' (Breiman and Cutler 2005). RF algorithm mentioned above works for dust detection as follows. For instance, if $N$ is chosen as 300 , the algorithm produces 300 trees meaning 300 different classification results for a particular pixel. Assuming that a pixel is classified as dust in 250 trees, as soil in 20 trees and is assigned to water class in 30 trees, the predicted output for this pixel will be dust. For this study, the ratio of number of trees labelled dust and overall trees should be higher than 0.7 to assign a pixel as dust.

In order to evaluate the significance of the features introduced in this work, the normalized feature importance is calculated. For each tree, the samples being OOB are permuted in the respective feature. They are used for the input of the trees and then, the corresponding accuracy is computed. The feature importance is the difference in prediction accuracy before and after permuting the sample. Let $\overline{\mathfrak{B}}^{(t)}$ be the OOB sample for a tree $t$, with $t \in\{1, \ldots, n t r e e\}$. The feature importance can be defined as (Breiman and Cutler 2008):

$$
\begin{aligned}
F I^{(t)}\left(x_{j}\right)= & \frac{\sum_{i \in \overline{\mathfrak{B}}^{(t)}} I\left(y_{i}=\widehat{y}_{i}^{(t)}\right)}{\left|\overline{\mathfrak{B}}^{(t)}\right|} \\
& -\frac{\sum_{i \in \overline{\mathfrak{B}}^{(t)}} I\left(y_{i}=\widehat{y}_{i, \pi_{j}}^{(t)}\right)}{\left|\overline{\mathfrak{B}}^{(t)}\right|}
\end{aligned}
$$

where $\widehat{y}_{i}^{(t)}=f^{(t)}\left(x_{i}\right)$ is the predcited classes for observation $i$ before and $\widehat{y}_{i}^{(t)}=f^{(t)}\left(x_{i}, \pi_{j}\right)$ is the predicted classes for observation $i$ after permuting its value of feature $j$. The average of differences of the accuracies of a feature is the raw importance for these features:

$$
F I\left(x_{j}\right)=\frac{\sum_{t=1}^{n \text { tree }} F I^{(t)}\left(x_{j}\right)}{n \text { tree }}
$$

Dividing the raw feature importance by the respective standard deviation results in the normalized feature importance. A high value means that a feature has a high importance for the entire RF.

\subsection{Method of evaluation}

Validation of dust detection algorithms is not a straightforward task. Unfortunately, there are no adequate 'ground truth' surface observations in the areas to test against; but we have two alternatives: (1) results are visually compared with OMAERUV AAOD product, and (2) small ROIs have been selected manually in order to assess the validity of the approaches using Probability Of False Detection (POFD) and Probability Of Missing Detection (POMD). The POFD can be defined as the fraction of forecasts of dust phenomenon accompanying with non-occurrences:

$$
\mathrm{POFD}=\frac{b}{(a+b)}
$$

where $a$ and $b$ are number of detected dusty pixels with occurrences and non-occurrences, respectively. POMD can be expressed as the ratio of underforecasting dusty pixels accompanying with occurrences:

$$
\mathrm{POMD}=\frac{c}{(a+c)}
$$

where $c$ is the number of ground truth dusty pixels that are not detected.

\section{Results and discussion}

\subsection{RF set-up}

For RF, it is necessary to train the classifiers with prototypes. The procedure of choosing training data can be done using site visits, prior knowledge and experience of the case study area, using maps, empirical threshold for indices such as NDVI or photointerpretation of colour composite product created from the image data. When the RF model is established with adequate and diverse prototypes, it can be used for other images; thus, it might be regarded as a semi-automatic approach. The main classes (if available) are cloud, dust, soil, snow, vegetation and water. In this study, we make use of Colour Infrared (CIR) colour (i.e., NIR as red, green light as blue and red light as green) for manually selecting vegetation, soil and water classes. Distinguishing snow and cloud and discriminating between dust and bright surfaces is a difficult task when using only a CIR or true-colour composite. Therefore, a new false-colour composite for separating dust from soil, and snow from cloud is proposed in this study. It is formed from bands 6 , 20 and 31. Since it is a single image and there exists low correlation between snow, dust and cloud, it is possible to create fully automatic approach in 

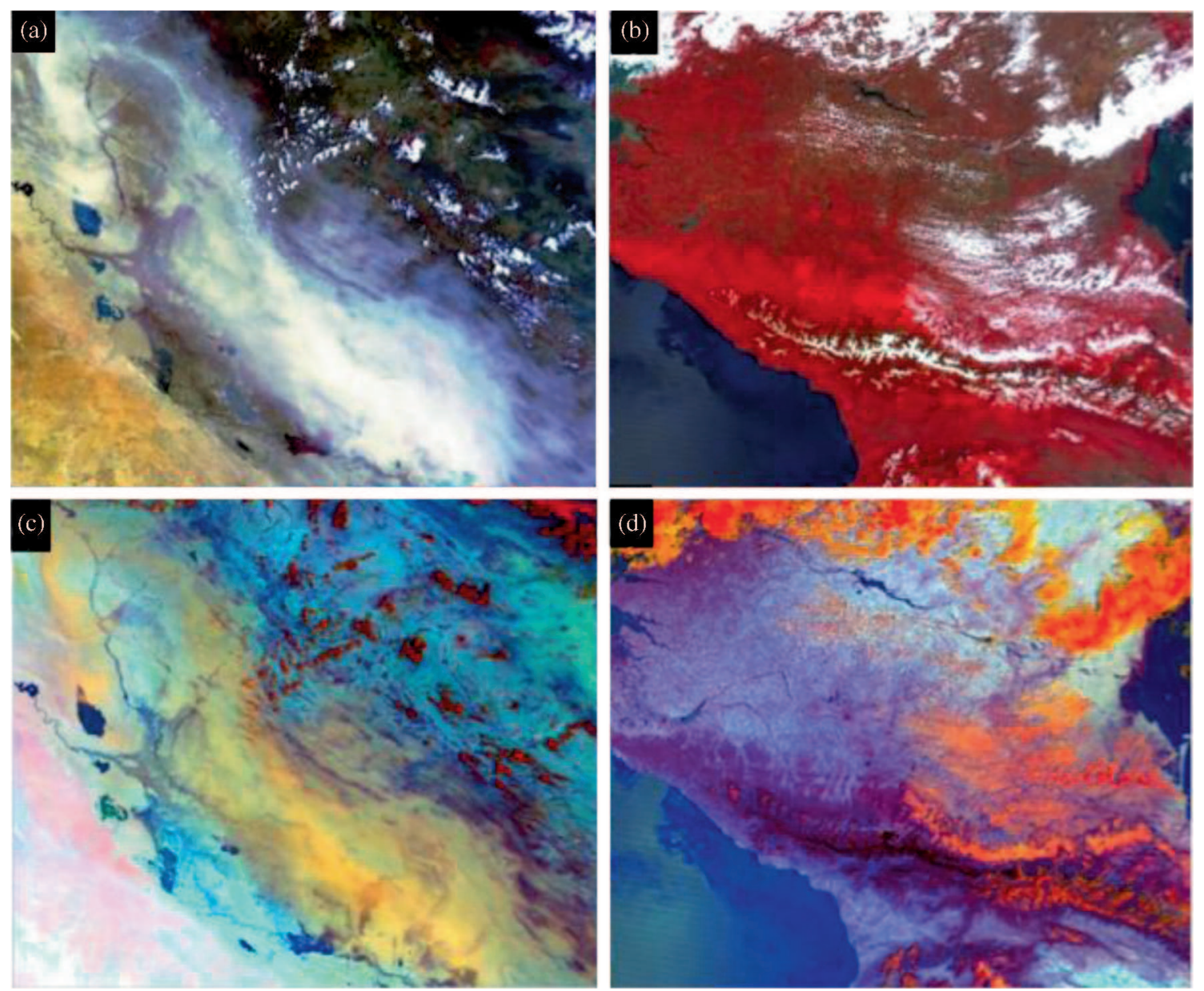

Figure 4. (a and b) The CIR false colour images of MODIS observations in dust and snow regions; it is problematic to separate snow from cloud and dust from cloud and soil, (c and d) the proposed false colour composite for better interpretation. Dust, snow and cloud are now more noticable.

future works using a5 colour-based segmentation method and stratified sampling. To illustrate the composite, two scenes are shown in figure 4 in two different false-colour composites (i.e., one CIR and another the proposed composite). The left scene shows a relatively bright thick dust over both bright surface and low height clouds. While in CIR colour composite, the difference between cloud, bright surface and dust is not discernable perfectly, the proposed colour composite demonstrates distinctive colors between three classes. In the right scene, the similarity between snow and cloud classes leads the classification to failure using only CIR colour composite. Conversely, the proposed composite depicts distinguishing colours among the classes.

The total sample pixels in each case study include approximately $0.01 \%$ of whole pixels. Jeffries-Matusita and Transformed Divergence are commonly used to determine the mathematical separability of classes or how well the selected ROI pairs are statistically separate (Richards and
Xiuping 2006). Both factors are close to 2.0 for all the ROI pairs in the case studies. As outlined before, the user-defined parameters in RF affecting directly the classification performance are $N$ and $m$. Satisfactory results obtained with $N$ and $m$ are equal to 200 and 3 (square root of 12 input features) respectively. The OOB error is unbiased and is a good estimation for the generalization error. The OOB accuracy of the eight case studies is provided in the supplementary material. Overall, the OOB accuracies are found convincing $(>0.99)$ for the selected RF inputs.

Figure 5 represents the mean normalized feature importance for the case studies. It demonstrates that the considered features contain significant information that are suitable for increasing the separability of dust plumes from other classes. Most important features are found for the visible and near-infrared bands, while $1.37 \mu \mathrm{m}$ shows the lowest feature importance due to strong water vapour absorption in this channel, indicating that this band is more suitable for cold and high height clouds than other classes. 


\subsection{Results of dust events}

The results of eight case studies on dust detection are presented in this section. When implementing Zhao's method, it was observed that a considerable ambiguity exists with regard to the threshold of BTD $(3.9$ and $11 \mu \mathrm{m})$. This test was used for identifying water clouds. However, many of the dusty pixels did not satisfy this condition and were then filtered out (figure 6). Therefore, comparing visually detected dusty pixels and the output of Zhao's method, we altered the threshold of BTD (3.9 and $11 \mu \mathrm{m})$ to 15 . This modification has also performed in Algorithm Theoretical Basis Document (ATBD) for GOES-R ABI.

The dust detection results for the eight case studies depicted in figures 7 and 8 are highlighted in red colour.

Regarding dust storm on 25/01/2005 event (figure 7a), raised dust particles are difficult to detect in the visible scene and these thin dust plumes are coupled with bright surfaces. The OMI result also reveals the presence of thin dust storms. One of the problems of the physical-based approaches lies in finding appropriate BTD of a

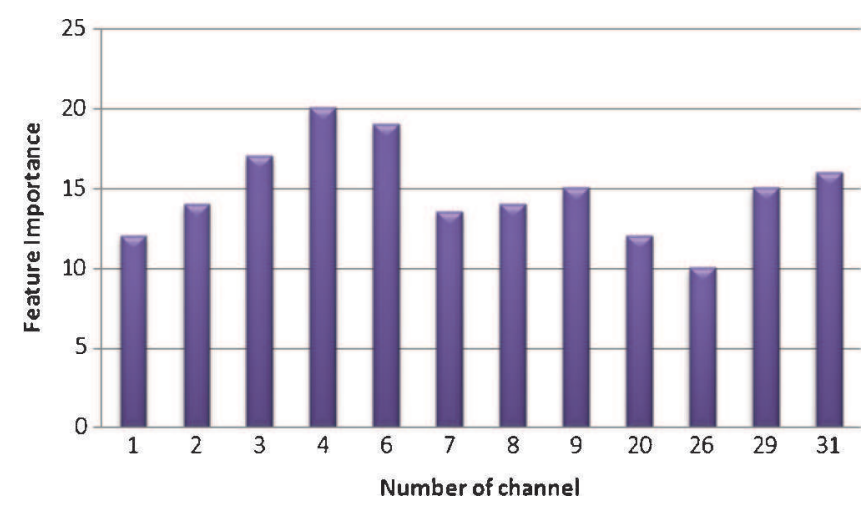

Figure 5. Average feature importance of eight case studies. shorter emissive band and a longer one. This weakness impedes introducing a unique threshold that fits every image. Lowering the BTD can reveal more dusty pixels; not only parts of thin dust storms are categorized as dust, but many pixels of bright surfaces also are labelled. This is again the reason for absence of dusty pixels on 17/09/2008 (figure 7b) and 25/01/2011 (figure 7c) events. BTD (12 and $11 \mu \mathrm{m}$ ) is so effective to identify thin cirrus clouds, because they generally have negative BTD (12 and $11 \mu \mathrm{m}$ ) (Ackerman 1997); that is why most of the cirrus clouds (white/gray over dust plumes in figure $7 \mathrm{a}$ and $\mathrm{d}$ ) have not been misidentified in both physical-based methods. The $\mathrm{RF}$ is very effective in detecting thin dust plumes and separating off cloudy pixels; particularly on the event on 11/05/2010 (figure $7 \mathrm{~d}$ ), thin dust plumes close to cirrus clouds have been detected precisely. In the event on 19/04/2005 (figure 8a), the dust plumes in the upper left of the scene are clearly visible. Although these dust particles should have been detected effortlessly, the physical-based approaches (particularly Zhao's method) did not identify pixels in the core of the dust plume. This is because of BTD (12 and $11 \mu \mathrm{m})$. Darmenov and Sokolik (2005) demonstrated that this BTD could change from 1 to even -0.5 for dust storms transported over oceans from seven different regions. The authors stated that the diversity of thresholds was likely due to different mineralogical composition. Given a lack of dust composition data, the BTD methods cannot be utilized to their full potential. In addition, some dust storms are caused by strong winds behind a cold front that leads to coexistence of dust particles with ice clouds. As outlined earlier, the BTD is highly sensitive to presence of upper cirrus cloud layer, even though heavy dust exists behind it. Due to this BTD, we also have some unidentified pixels in dust plumes located on left part of the scene on 20/06/2012

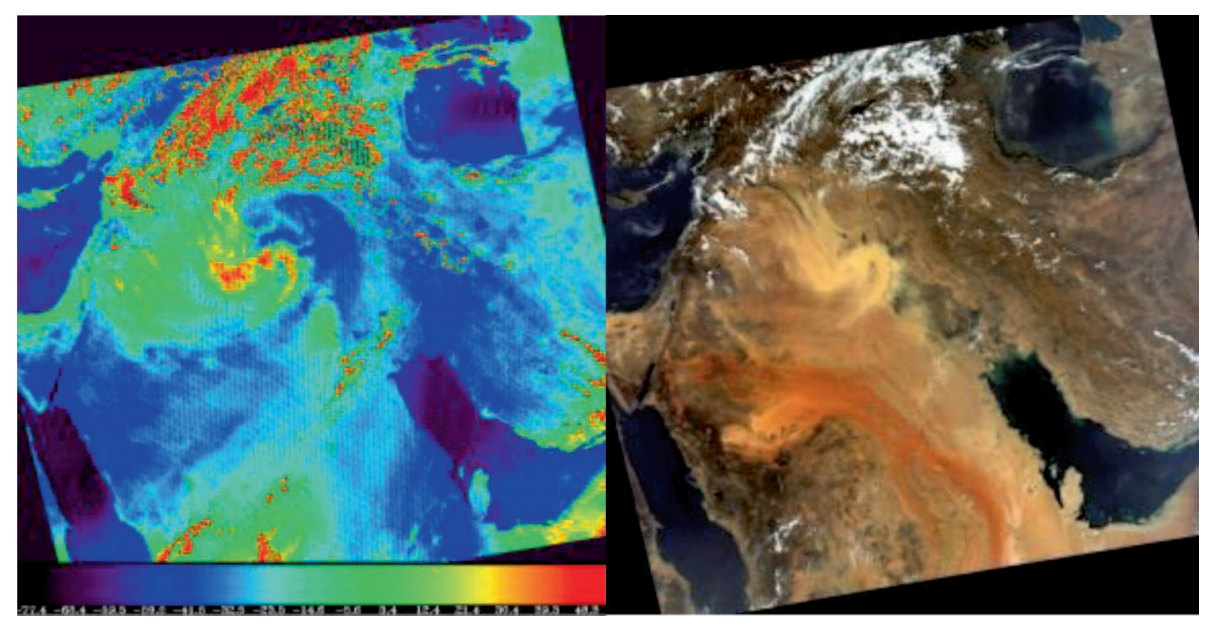

Figure 6. Left: BTD $(3.9,11 \mu \mathrm{m})$, right: RGB colour composite. 

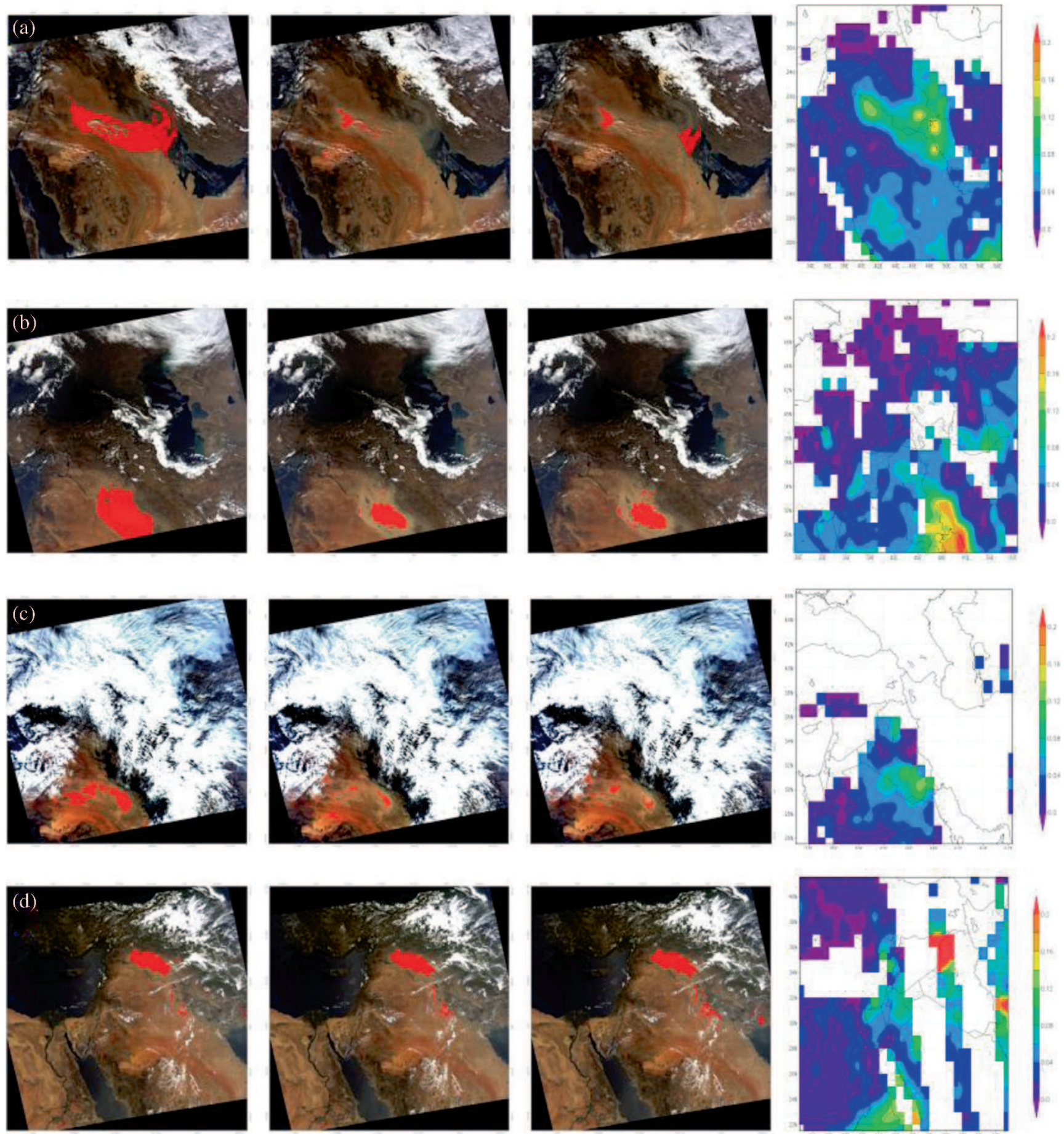

Figure 7. Dust storms on (a) 25/01/2005, (b) 17/09/2008, (c) 25/01/2010, and (d) 11/05/2010. From left to right: The result of RF, Xie's method, the result of Zhao's method and AAOD OMI product.

event (figure $8 \mathrm{~b}$ ). Accordingly, it is very difficult to detect dust under cirrus areas or dust-cloud mixed pixels. The RF result for this event certainly has not been influenced by the presence of thin clouds. Where the cloud cover is opaque, reflective bands are found to be visually very helpful to bring out heavy dust plumes. Therefore, this is a recognized advantage of taking into account these bands for classification-based approaches. We may be interested in knowing a confidence value for each pixel's labeled dust, because dust-cloud mixed pixels are subject to many uncertainties in determining aerosol properties. In RF, the probability output can be easily obtained for each pixel by dividing number of trees labelled dusty region with overall trees. The output has an advantage that makes it well suited to represent the probability of dust in a pixel. Figure 9 provides a clear picture of the RGB composite, the BTD and the probability of the dust storm on 19/04/2005. The correlation 

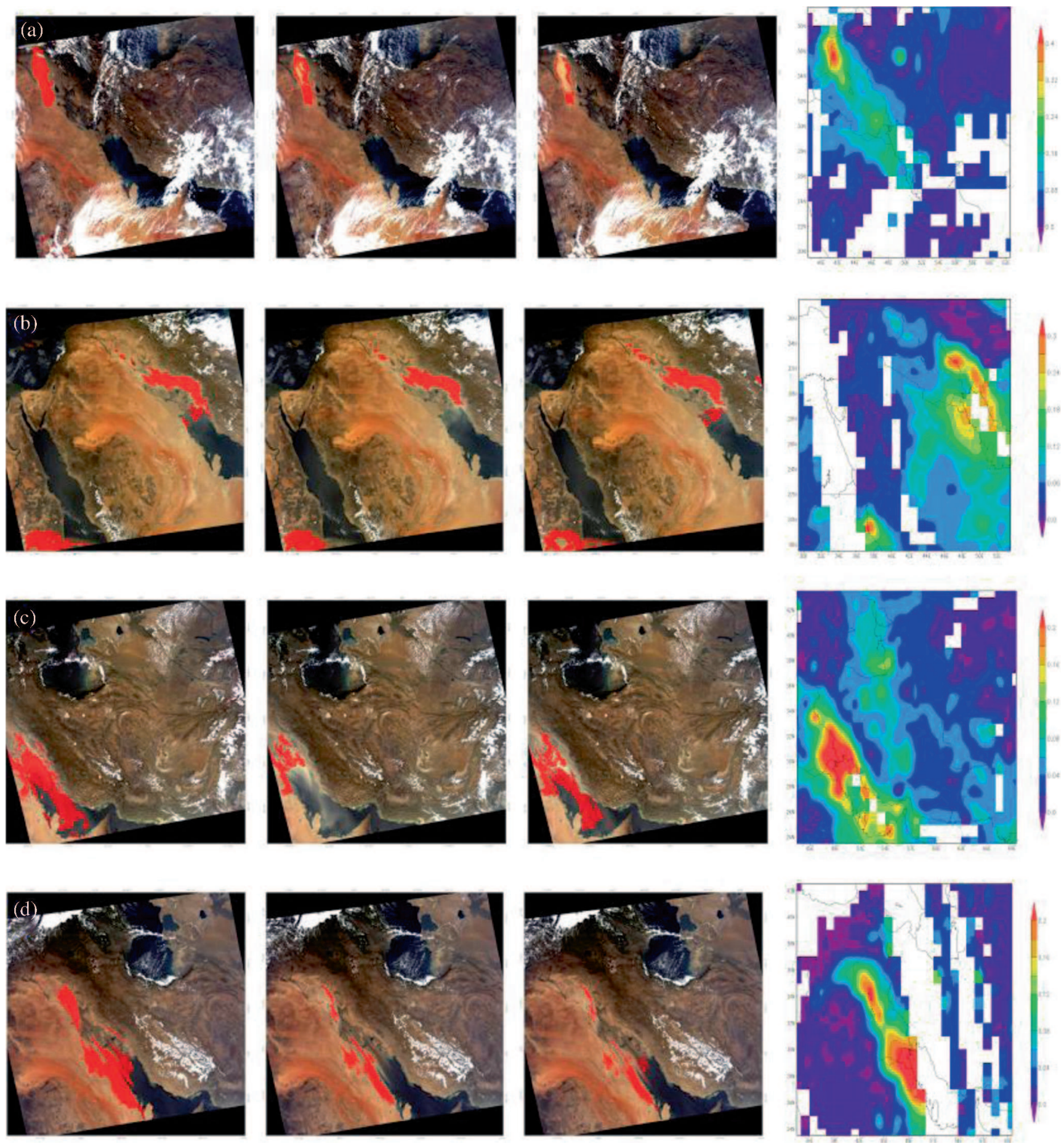

Figure 8. Dust storms on (a) 19/04/2005, (b) 20/06/2012, (c) 05/07/2005, and (d) 30/06/2011. From left to right: The result of RF, Xie's method, the result of Zhao's method and AAOD OMI product.

coefficient between the BTD and the probability of dust retrieved from $\mathrm{RF}$ is found 0.74 supporting the efficient statistical method used.

The event on $05 / 07 / 2005$ (figure 8c) was an extensive dust storm over both land and water. Once more, BTD $(12$ and $11 \mu \mathrm{m})$ is the main reason for some dissimilarities between the methods. Both $\mathrm{RF}$ and Zhao's method are effective in finding dust plumes over water due to distinct texture and luminance of mineral aerosols, whereas Xie's method has not been designed for this purpose. The final event on 30/06/2011 (figure 8d) shows a peculiarity which might be of interest. The peculiarity is the appearance of a dark brown dust plume (see figure 10). The physical-based approaches' outputs have confused this thick dust with surface. Since the methods are preferably looking for bright dust plumes, the tests using reflective bands (such as MNDVI in Zhao's and $\log$ (red) in Xie's method) completely have neglected this kind of dust storms. 


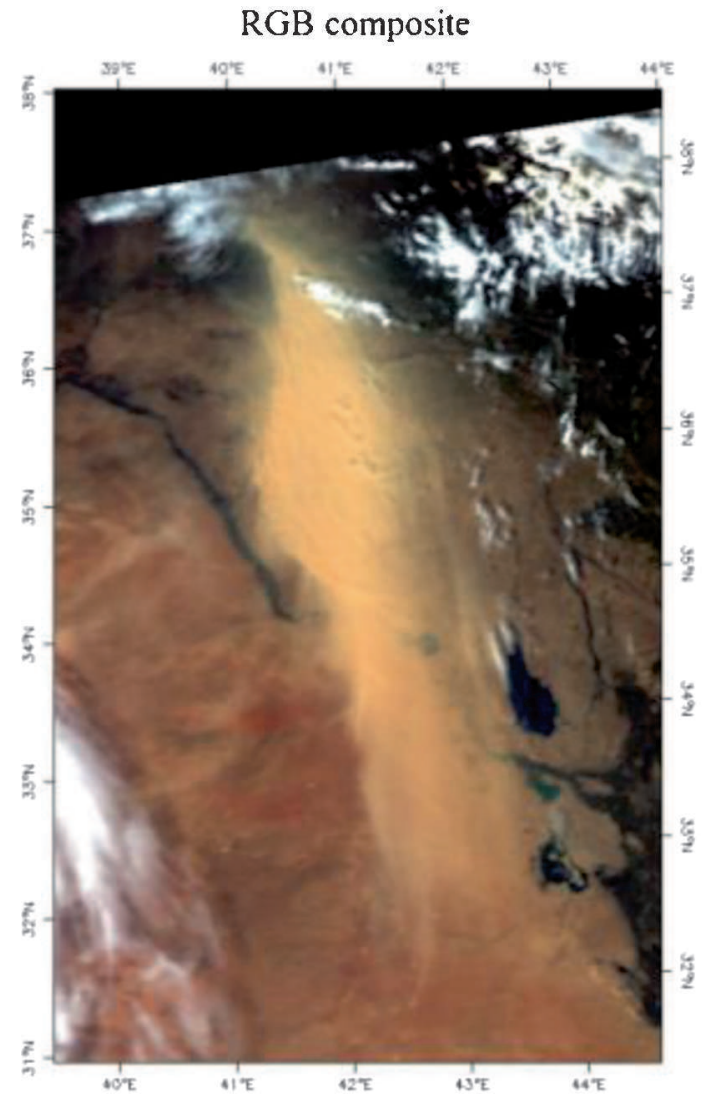

The probability of dust class

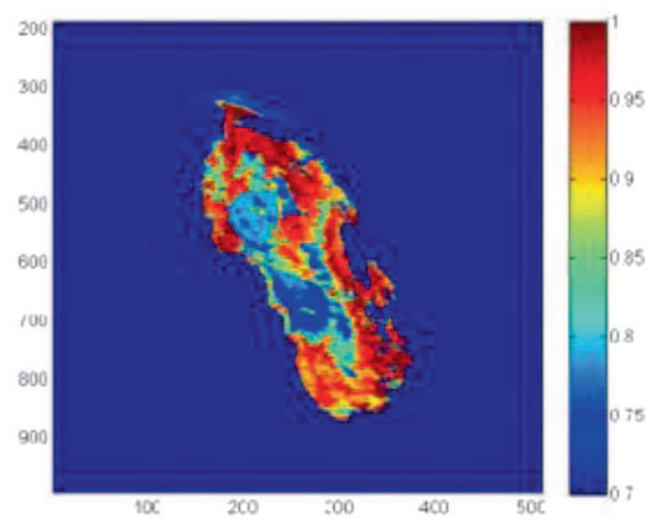

BT12-BT11

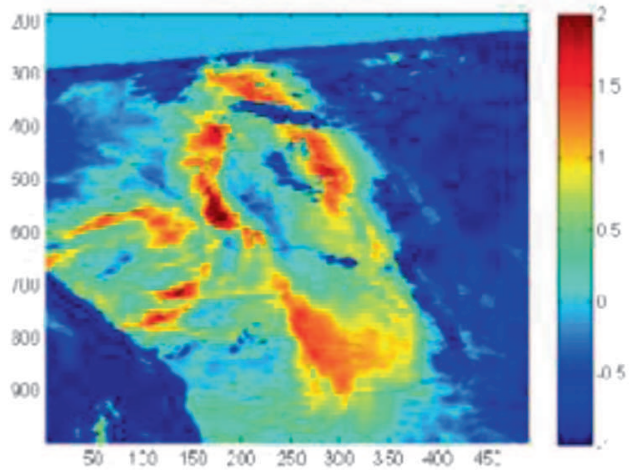

Figure 9. MODIS RGB image (left) and the corresponding probability of dust class and BTD $(12,11 \mu \mathrm{m})$.

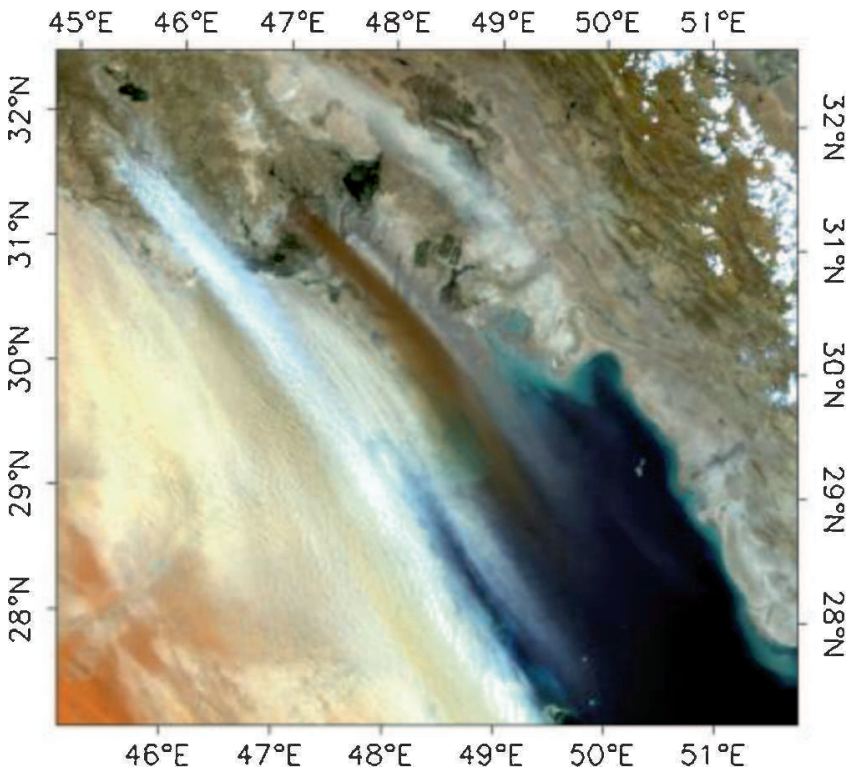

Figure 10. Dark brown dust plumes on 30/06/2011.

It is not an easy task to tune reflective tests for this specific event, and it undoubtedly needs extra efforts. RF shows little confusion of both bright and dark dust storms with ground surfaces due to considering deployed effective features and prototypes.
Table 2. Results of the methods' accuracy.

\begin{tabular}{lcc}
\hline Method & POMD & POFD \\
\hline Zhao's method & 0.21 & 0.08 \\
Xie's method & 0.46 & 0.14 \\
Random Forests & 0.07 & 0.06 \\
\hline
\end{tabular}

In order to quantify the accuracy of the results, POFD and POMD were calculated by comparing the resultant dusty pixels and manually selected ROIs. Table 2 lists the results of the statistical metrics.

According to the results obtained, this study shows good capability of RF on dust detection over both water and land simultaneously. This classifier offers a potential way to detect even dusty regions with relatively low optical depth and close to cloud intensity. When we repeat the calculation of POFD and POMD, with the exception of Xie's method, lower POFD and POMD are found over water than those over land (not shown) due to the relative lower, spectrally flat and more uniform surface. It should be noted that dead pixels affect physicalbased results. Conversely, RF is not sensitive to this situation. Bad pixels in few bands do not impair overall performance significantly. Despite 
of attaining fair accuracy, Xie's method has not been designed to detect dusty regions over water. A serious criticism of Xie's method is regarding pixels with negative values of BTD (11 and $12 \mu \mathrm{m})$ as cloud, since lower clouds can show positive BTD (11 and $12 \mu \mathrm{m})$. Although Zhao's method contributed to introducing dust-related features, it shows worse performance than RF for detection of thin dust over land or covered by optically thin cloudy pixels.

\section{Conclusions}

The aim of this study was to evaluate dust detection approaches in MODIS imagery. Two physicalbased methods and RF were used and the resultant dust class was compared to OMI AAOD product. Using several case studies in the Middle East, it was shown that RF captures the dust events with good performance metrics. Due to the fact that $\mathrm{RF}$ considered different mineralogical dust compositions in different underlying surfaces, satisfactory results were obtained compared to the physicalbased methods which truly had no universal thresholds. This indicated that RF possessed both generalization and robustness characteristics. Another advantage of making use of $\mathrm{RF}$ is undemanding auxiliary data such as cloud mask, because clouds were regarded as a class. This class can be compared to MOD35 product in future works. Nevertheless, the proposed method had some limitations such as manually collecting prototypes for training RF. This can be developed to an automatic process using segmentation in future works. Because AOT product is produced two days after acquiring time, and it has $10 \mathrm{~km}$ pixel dimension, dust class retrieved by $\mathrm{RF}$ developed by automatically collecting prototypes can be an appropriate complementary for AOT MODIS product in case of dust storm forecasting and warning system.

The proposed approach for detecting dust aerosols could be developed with further studies including: (1) creating fully automatic approach using segmentation in order to train RF, (2) enhancing the algorithm by combining CALIPSO and fusion by ASTER data, (3) validation of the method with only emissive bands in night-time, and (4) evaluation of resultant cloud class with MOD35.

\section{Acknowledgement}

The authors would like to thank M R Saradjian and $\mathrm{P}$ Ciren for their constructive comments that improved significantly the quality of the manuscript.

\section{References}

Ackerman S A 1989 Using the radiative temperature difference at $3.7 \mu \mathrm{m}$ and $11 \mu \mathrm{m}$ to trace dust outbreaks; Remote Sens. Environ. 27(2) 129-133.

Ackerman S A 1997 Remote sensing aerosols using satellite infrared observations; J. Geophys. Res. 102 17,069-17,079.

Alpert P, Kishcha P, Shtivelman A, Krichak S O and Joseph J H 2004 Vertical distribution of Saharan dust based on 2.5-year model predictions; Atmos. Res. 70 109-130.

Badarinath K V S, Kumar Kharol S, Kaskaoutis D G and Kambezidis H D 2007 Dust storm over Indian region and its impact on the ground reaching solar radiation: A case study using multi-satellite data and ground measurements; Sci. Total Environ. 384 316-332.

Breiman L 1996 Bagging predictors; Machine Learning 24 123-140.

Breiman L 2001 Random Forests; Machine Learning 45(1) $5-32$.

Breiman L 2002 Manual on Setting Up, Using, and Understanding Random Forests V3.1; Available at http://oz. berkeley.edu/users/breiman/Using_random_forests_V3.1. pdf.

Breiman L and Cutler A 2005 Random Forest; Available at:

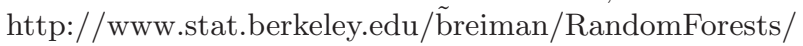
cc_home.htm.

Breiman L and Cutler A 2008 Random forests-Classification manual; http://www.math.usu.edu/ adele/forests/.

Chacon-Murguía M I, Quezada-Holguín Y, Rivas-Perea P and Cabrera S 2011 Dust storm detection using a neural network with uncertainty and ambiguity output analysis; Pattern Recognition, Lecture Notes in Computer Science 6718 305-313.

Darmenov A and Sokolik I N 2005 Identifying the regional thermal-IR radiative signature of mineral dust with MODIS; Geophys. Res. Lett. 32 L16803.

Efron B and Tibshirani R 1993 An Introduction to the Bootstrap; Chapman \& Hall Publisher.

Evan A T, Heidinger A K and Pavolonis M J 2006 Development of a new over-water Advanced Very High Resolution Radiometer dust detection algorithm; Int. J. Remote Sens. 27(18) 3903-3924.

Freund Y and Schapire R E 1997 A decision-theoretic generalization of on-line learning and an application to boosting; J. Comput. Syst. Sci. 55(1) 119-139.

Frey R A, Ackerman S A, Liu Y, Strabala K I, Zhang H, Key J and Wang X 2008 Cloud Detection with MODIS, Part I: Recent Improvements in the MODIS Cloud Mask; J. Atmos. Oceanic Technol. 25 1057-1072.

Friedman J, Hastie T and Tibshirani R 1998 Additive logistic regression: A statistical view of boosting; Ann. Statist. 28 337-407.

Gao B C, Yang P, Han W, Li R R and Wiscombe W J 2002 An algorithm using visible and 1.38-m channels to retrieve cirrus cloud reflectances from aircraft and satellite data; IEEE Trans. Geosci. Remote Sens. 40 1659-1668.

Gislason P O, Benediktsson J A and Sveinsson J R 2006 Random forest for land cover classification; Pattern Recognition Lett. 27 294-300.

Hao X and Qu J J 2007 Saharan dust storm detection using moderate resolution imaging Spectroradiometer thermal infrared bands; J. Appl. Remote Sens. 1013510.

Hsu N C, Tsay S, King M D and Herman J R 2004 Aerosol properties over bright-reflecting source regions; IEEE Trans. Geosci. Remote Sens. 42(3) $557-569$.

James T K, Chuang C C and Anthony S W 2007 Influence of dust composition on cloud droplet formation; Atmos. Environ. 41(14) 2904-2916. 
Kaskaoutis D G, Kambezidis H D, Nastos P T and Kosmopoulos P G 2008 Study on an intense dust storm over Greece; Atmos. Environ. 42(9) 6884-6896.

Liaw A and Wiener M 2002 Classification and regression by Random Forest; $R$ news 2(3) 18-22.

MacKinnon D J, Chavez P S, Fraser R S, Niemeyer T C and Gillette D A 1996 Calibration of GOES-VISSR, visibleband satellite data and its application to the analysis of a dust storm at Owens Lake, California; Geomorphology $17229-250$.

Martins J V, Tanré D, Remer L, Kaufman Y, Mattoo S and Levy R 2002 MODIS cloud screening for remote sensing of aerosols over oceans using spatial variability; Geophys. Res. Lett. 29(12) MOD4-1.

McGowan HA and Clark A 2008 A vertical profile of PM10 dust concentrations measured during a regional dust event identified by MODIS Terra, western Queensland, Australia; J. Geophys. Res. 113 F02S03.

Mie G 1908 Beitrage zur Optik truber Medien, speziell kolloida-ler Metallosungen; Ann. Phys. 25 377-446.

Moorthy K K, Babu S S, Satheesh S K, Srinivasan J and Dutt C B S 2007 Dust absorption over the 'Great Indian Desert' inferred using ground-based and satellite remote sensing; J. Geophys. Res. 112(D9) D09206.

Nicholson S E 2000 Land surface processes and Sahel climate; Rev. Geophys. 38(1) 117-139.

Qu J J and Kafatos M 2006 Asian dust storm monitoring combining Terra and Aqua MODIS SRB measurements; Geosci. Remote Sens. Lett. 3(4) 484-486.

Raileanu L E and Stoffel K 2004 Theoretical comparison between the Gini Index and Information Gain criteria; Ann. Math. Art. Intell. 41 77-93.

Rees 2001 Physical Principles of Remote Sensing; Cambridge University Press.

Richards J A and Xiuping J 2006 Remote Sensing Digital Image Analysis; Springer-Verlag, Berlin, Heidelberg, New York.
Rivas-Perea P, Rosiles J G, Chacon Murguia M I and Tilton J J 2010 Automatic dust storm detection based on supervised classification of multispectral data; Soft Computing for Recognition Based on Biometrics, Studies Comput. Intell. 312 443-454.

Roskovensky J K and Liou K N 2005 Differentiating airborne dust from cirrus cloud using MODIS data; Geophys. Res. Lett. 32 L12809.

Rudin C, Daubechies I and Schapire R E 2004 The dynamics of adaboost: Cyclic behavior and convergence of margins; J. Machine Learning Res. 5 1557-1595.

Salomonson V V, Barnes W L, Maymon P W, Montgomery $\mathrm{H} \mathrm{E}$ and Ostrow H 1989 MODIS: advanced facility instrument for studies of the earth as a system; IEEE Trans. Geosci. Remote Sens. 27 145-153.

Torres O, Tanskanen A, Veihelmann B, Ahn C, Braak R and Bhartia P K 2007 Aerosols and surface UV products from Ozone Monitoring Instrument observations: An overview; J. Geophys. Res. 112(D4).

Yang $\mathrm{P}$ et al. 2003 The spectral signature of mixed-phase clouds composed of non-spherical ice crystals and spherical liquid droplets in the terrestrial window region; $J$. Quant. Spectr. Rad. Trans. 79 1171-1188.

Xie Y 2009 Detection of Smoke and Dust Aerosols Using Multi-sensor Satellite Remote Sensing Measurements; Dissertation of Doctor of philosophy at George Mason University.

Zha Y and Li L 2007 Influence of the 17 April 2006 dust storm on Moderate Resolution Imaging Spectroradiometer data for land cover identification; J. Geophys. Res. 112 D14205.

Zhao T X P, Ackerman S A and Guo W 2010 Dust and smoke detection for multi-channel imagers; Remote Sens. 2 2347-2368.

Zhu A, Ramanathan V, Li F and Kim D 2007 Dust plumes over the Pacific, Indian and Atlantic Oceans: Climatology and radiative impact; J. Geophys. Res: Atmos. 112(D16) D16208. 